\title{
Transparenz allein reicht bei Interessenkonflikten nicht aus - Pro
}

\author{
Transparency Alone is not Sufficient for the Management of Conflicts \\ of Interest - Pro
}

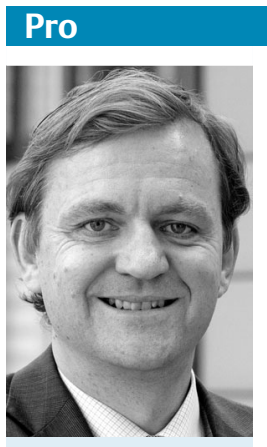

Klaus Lieb
Am 30.9.2014 ist die bisher größte Transparenzinitiative zur Offenlegung von finanziellen Interessenkonflikten bei US-amerikanischen Ärzten online gegangen [1]. Über eine allgemein zugängliche Website (www.cms. gov/openpayments/)

sind alle Zahlungen pharmazeutischer Unternehmen und Medizinproduktehersteller an US-amerikanische Ärzte öffentlich gemacht worden. Grundlage dieser Transparenz ist ein 2007 initiiertes und 2012 verabschiedetes US-amerikanisches Gesetz, der „Physician Payment Sunshine Act (PPSA)“, nach dem pharmazeutische Unternehmen und Medizinproduktehersteller alle Zahlungen an Ärzte und Lehrkrankenhäuser an die Centers for Medicare \& Medicaid services (CMS) zu melden haben. Die Zahlungen werden auf der Website in den 3 Kategorien allgemeine Zahlungen (z.B. Essenseinladungen, CME-Sponsoring, Reisekostenerstattungen usw.), Zahlungen für Forschungsaktivitäten und Beteiligungen (z. B. Lizenzzahlungen) transparent gemacht.

Am 30.9.2014 wurden alle Zahlungen von August-Dezember 2013 öffentlich gemacht, für Juni 2015 ist die Offenlegung aller Zahlungen für das Jahr 2014 vorgesehen. Demnach wurden für die 5 Monate in 2013 etwa 3,5 Mrd. US\$ an 546000 Ärzte und 1360 Lehrkrankenhäuser gezahlt [2]. Entsprechend den Angaben auf der Website sind die 5 Spitzenverdiener Orthopäden, die aus Lizenzen in der Spitze 7,3 Mio. US\$ erzielt hatten. An die Berufsgruppe der Psychiater (Rang 4) wurden insgesamt ca. 18,7 Mio. US\$ gezahlt. Unter den Zahlungen waren auch 5,8 Mio. US\$ Sponsoring (ohne Lizenzgebühren) für Lurasidon (Latuda ${ }^{\circledR}$ ), ein neues Antipsychotikum, das 2010 in den USA und 2014 in Europa durch die EMA zugelassen wur- de (in Deutschland steht die frühe Nutzenbewertung an).

Eine weitere aktuelle Transparenzinitiative geht vom Verband forschender Arzneimittelhersteller (VfA) aus. Entsprechend der Kodices der „Freiwilligen Selbstkontrolle für die Arzneimittelindustrie e.V.“ (FSA) werden bereits heute schon Zahlungen der 37 Mitgliedsunternehmen an Selbsthilfegruppen auf einer Website [3] veröffentlicht. Für 2016 ist geplant, alle Zahlungen an Ärzte auf den jeweiligen Websites der Mitgliedsunternehmen rückwirkend für 2015 offenzulegen.

Die Vorteile des PPSAs gegenüber der geplanten Transparenz der FSA liegen darin, dass die Transparenz in einem Gesetz festgelegt und damit verpflichtend ist, während bei der FSA die Offenlegungen durch die Industrie freiwillig sind und jeder Arzt der Veröffentlichung vorher schriftlich zustimmen muss. Gibt er sein Einverständnis nicht, werden die Daten nur anonymisiert veröffentlicht. Ein weiterer Vorteil des PPSA ist, dass die Daten aggregiert für alle Firmen auf einer einzigen Website dargestellt werden, die einfach nach $\mathrm{Na}$ men zu durchsuchen ist. Die FSA plant dagegen, die Informationen nicht in einer Website zusammenzuführen, sondern auf die Website jedes Mitgliedsunternehmens zu stellen. Dies wird die Suche sehr mühsam machen, auch wenn geplant ist, die Websites in ihrem Aufbau zu vereinheitlichen. Ein weiterer Vorteil des PPSA ist, dass Zahlungen aller pharmazeutischen Unternehmen und Medizinproduktehersteller veröffentlicht werden, während das bei der FSA nur die (relativ) wenigen Mitgliedsunternehmen des VfA tun. Die Nichtmeldung wird beim PPSA mit hohen Strafen für die Firmen geahndet, während die Strafzahlungen bei der FSA vage bleiben.

Beide Initiativen haben weitere Nachteile: Durch die Zustimmungspflicht bei der FSA ist zu erwarten, dass es zu begrenzter Transparenz kommt, da wahrscheinlich gerade die Ärzte mit vielen Interessenkonflikten deren Veröffentlichung nicht zustimmen werden. Aber auch beim PPSA wurden 40\% der Daten wegen möglicher Inkorrektheiten der Daten (die Prüfzeit für die Ärzte war sehr kurz) vorläufig anonymisiert veröffentlicht. Darüber hinaus werden nur finanzielle Interessenkonflikte offengelegt, obwohl bekannt ist, dass auch nichtfinanzielle Interessenkonflikte (z.B. die aktive Mitarbeit in einem Berufsverband oder die ideelle Vertretung einer Therapieschule) relevante Interessenkonflikt-Konstellationen begründen. Die geschätzten Kosten für die Umsetzung des PPSA liegen mit 269 Mio. \$ im 1. Jahr und 180 Mio. \$ für jedes weitere Jahr extrem hoch [4], Zahlen zu den Kosten der FSA liegen bisher nicht vor.

Selbstverständlich ist Transparenz die Voraussetzung dafür, Interessenkonflikte überhaupt wahrzunehmen und damit umzugehen. Insofern wäre ein PPSA für Deutschland zu begrüßen, der im Gegensatz zu der nicht ausreichenden Transparenz der FSA Klarheit für die Situation in Deutschland schaffen würde. Allerdings müssen bei diesen Überlegungen folgende Aspekte bedacht werden: Erstens wären die Kosten für eine solche Transparenz extrem hoch - hier stellt sich die Frage, ob dieses Geld nicht besser an anderer Stelle, z.B. für unabhängige Fortbildung, für die Unterstützung der Ärzte für eigene Transparenz in der Praxis, für die finanzielle Unterstützung unabhängiger Gremien zur Arzneimittelbewertung oder Leitliniengruppen eingesetzt werden könnte? Zweitens würde man durch ein solches Transparenzgesetz viel Zeit zur Regulierung von Interessenkonflikten verlieren. Denn bedenkt man, dass die Gesetzesinitiative in den USA 2007 startete, würden alle Aktivitäten zur Reduktion bzw. zur Regulierung von Interessenkonflikten auf die lange Bank geschoben, nach dem Motto: Jetzt schaffen wir erst mal Transparenz, und dann überlegen wir uns, was wir damit machen. Drittens kann Transparenz aufseiten der Patienten nur mit einem ausreichenden Problembewusstsein und Vorwissen bewertet werden, an- 
sonsten sind unintendierte und möglicherweise negative Auswirkungen auf das Vertrauen in die Ärzteschaft nicht unwahrscheinlich. Das spricht dafür, dass Transparenz nie für sich allein ausreichend ist, sondern immer mit Informationsvermittlung kombiniert werden muss.

Das Wichtigste aber ist, dass sich die Auswirkungen von Interessenkonflikten durch alleinige Transparenz nicht beseitigen lassen. Interessenkonflikte sind definiert als Situationen, die ein Risiko schaffen, dass professionelles Urteilsvermögen oder Handeln, welches sich auf ein primäres Interesse (hier: Das Beste für den Patienten zu tun) bezieht, durch sekundäre Interessen (hier: Beziehungen zur Industrie) unangemessen beeinflusst wird [5]. Das Problem sind daher nicht in erster Linie die Interessenkonflikte selbst, sondern das beeinflusste bzw. verzerrte Urteilsvermögen oder Handeln („bias“) dessen, der Interessenkonflikte hat. Oder mit anderen Worten: Interessenkonflikte sind nicht per se schlecht oder verwerflich, sie können aber durch ihren Einfluss auf Urteilen und Handeln schädliche Auswirkungen haben. Daraus wird auch leicht nachvollziehbar, dass Transparenz von Interessenkonflikten allein nicht das eigentliche Problem, nämlich den „bias“ beseitigt, dessen Auftretenswahrscheinlichkeit durch Interessenkonflikte erhöht wird. Je enger nun die Beziehungen zu einem pharmazeutischen Unternehmen sind, desto höher ist in der Regel die Gefahr des „bias“. So kann das Handeln eines Arztes, der infolge des Besuchs einer gesponserten Fortbildungsveranstaltung die Medikamente des einladenden pharmazeutischen Unternehmens bevorzugt verschreibt, obwohl andere ggf. preisgünstiger, wirkungsvoller und sicherer sind [6, 7], für den einzelnen Patienten Schaden anrichten, während das Urteil eines Mitglieds einer Leitlinienkommission, wenn es regelmäßig Vorträge auf Satellitensymposien eines Unternehmens hält [8] und so die Leitliniengestaltung zugunsten eines pharmazeutischen Produktes beeinflusst, im Prinzip alle Patienten mit dem entsprechenden Krankheitsbild schädigen. Solcher „bias“ lässt sich nicht allein durch Transparenz reduzieren, sondern nur, wenn die Interessenkonflikte selbst reduziert werden und, falls nicht möglich, der Umgang mit ihnen reguliert wird.

In Anlehnung an das Vorgehen des amerikanischen Institute of Medicine [9] hat die Arzneimittelkommission der deutschen Ärzteschaft (AkdÄ) 2011 ein Formblatt zur Erfassung von Interessenkonflikten ihrer Mitglieder entwickelt [10]. Seit März bzw. November 2014 sind alle Interessenkonflikte der derzeit 37 ordentlichen und fast 150 außerordentlichen Mitglieder der Jahre 2010-2013 auf der Website der AkdÄ einzusehen. Für die ordentlichen Mitglieder werden 2015 und für die außerordentlichen Mitglieder 2016 auch die genauen Honorare für das jeweils vorangehende Jahr offengelegt. Seit 2012 haben die Mitglieder begonnen, ihre Interessenkonflikte zu reduzieren, und Mitglieder mit Interessenkonflikten können seit 2013 bei der Bewertung von Arzneimitteln nur noch in begrenztem Maße mitarbeiten. Entsprechend dieser Regeln muss der Hauptverantwortliche einer Stellungnahme seit 3 Jahren frei von Interessenkonflikten in Bezug auf das zu bewertende Arzneimittel und Konkurrenzprodukte sein, in der Arbeitsgruppe dürfen nur max. ein Drittel der Experten Interessenkonflikte haben, und diese wiederum dürfen auch keine Texte vorformulieren. Mitglieder mit sehr engen Marketingbeziehungen (z.B. Mitglieder eines speaker's bureaus) sind grundsätzlich bei Bewertungen auszuschließen. Wie wichtig die Unabhängigkeit der Kommission ist, ergibt sich daraus, dass die AkdÄ neben ihrem zahnärztlichen Pendant die einzige Kommission ist, die auf gesetzlicher Grundlage in die frühe Nutzenbewertung neuer Arzneimittel im Rahmen des AMNOG-Verfahrens einbezogen ist.

Zusammengefasst ist Transparenz nur ein erster wichtiger Schritt zu mehr Unabhängigkeit von Ärzten und Wissenschaftlern, der gefolgt werden muss von weiteren Maßnahmen zur Reduktion von Interessenkonflikten und zum adäquaten Umgang mit ihnen. Dies alles sind wichtige
Maßnahmen, um das Vertrauen der Bevölkerung in die Ärzteschaft und in ein integres Gesundheitswesen zu erhalten.

\section{Literatur}

1 Der Physician Payment Sunshine Act in den USA - Erste Ergebnisse. Der Arzneimittelbrief 2014; 48: 88DB01

2 Jarvies $D$, Coombes $R$, Stahl-Timmins W. Open payments goes live with pharma to doctor fee data: first analysis. BMJ 2014; 349: g6003

3 www.fs-arzneimittelindustrie.de

4 Health Policy Brief: The Physicians Payment Sunshine Act. Health Affairs October 2; 2014

5 Thompson DF. The challenge of conflict of interest in medicine. Z Evid Fortbild Qual Gesundhwes 2009; 103: 136-40

6 Spurling GK, Mansfield PR, Montgomery BD et al. Information from pharmaceutical companies and the quality, quantity, and cost of physicians' prescribing: a systematic review. PLoS Med 2010; 7: e1000352

7 Lieb K, Scheurich A. Contact between Doctors and the Pharmaceutical Industry, Their Perceptions, and the Effects on Prescribing Habits. PLoS One 2014; 9: e110130

8 Schott G, Dünnweber C, Mühlbauer B et al. Does the pharmaceutical industry influence guidelines? Two examples from Germany. Dtsch Arztebl Int 2013; 110: 575-583

9 Lo B, Field MJ, Hrsg. Conflict of interest in medical research, education and practice. 1 . Aufl. Washington DC: National Academies Press; 2009

10 Fachausschuss Transparenz und Unabhängigkeit der Bundesärztekammer bei der AkdÄ. www.akdae.de/Kommission/Organi sation/Mitglieder/Fachausschuesse/Trans parenz/index.html

Sie haben eine eigene Meinung zu diesem

Thema? Dann schreiben Sie uns an:

psychiat-praxis@thieme.de!

Korrespondenzadresse

Univ.-Prof. Dr. Klaus Lieb

Direktor der Klinik für Psychiatrie und

Psychotherapie, Universitätsmedizin Mainz

Untere Zahlbacher Straße 8

55131 Mainz

klaus.lieb@unimedizin-mainz.de

\section{Bibliografie}

DOI http://dx.doi.org/

10.1055/s-0034-1387519

Psychiat Prax 2015; 42: 12-13

(c) Georg Thieme Verlag KG

Stuttgart · New York

ISSN 0303-4259 